Bull. Austral. Math. Soc.

$16 \mathrm{~s} 90,16 \mathrm{D} 50$

VoL. 75 (2007) [127-133]

\title{
CHANGE OF RING AND TORSION-THEORETIC INJECTIVITY
}

\author{
Iuliu Crivei, Septimiu Crivei and Ioan Purdea
}

Let $\tau$ be a hereditary torsion theory in $R$-Mod. Then any ring homomorphism $\gamma$ : $R \rightarrow S$ induces in $S$-Mod a torsion theory $\sigma$ given by the condition that a left $S$-module is $\sigma$-torsion if and only if it is $\tau$-torsion as a left $R$-module. We show that if $\gamma: R \rightarrow S$ is a ring epimorphism and $A$ is a $\tau$-injective left $R$-module, then $\operatorname{Ann}_{A} \operatorname{Ker}(\gamma)$ is $\sigma$-injective as a left $S$-module. As a consequence, we relate $\tau$-injectivity and $\sigma$-injectivity, and we give some applications.

\section{INTRODUCTION}

A classical result states that if $I$ is a two-sided ideal of a ring $R$ and $E$ is an injective left $R$-module, then $\operatorname{Ann}_{E} I$ is injective as a left $R / I$-module (for instance, see [8, Proposition 2.27]). In this note we generalise it for injectivity with respect to a hereditary torsion theory. As a consequence we are able to deal with the problem of the behaviour of torsion-theoretic injectivity under the action of a ring homomorphism $\gamma: R \rightarrow S$. Under certain conditions we pass from relative injectivity in $R$-Mod to relative injectivity in $S$-Mod and we deal with the converse. The latter was previously discussed by several authors, for instance, Golan [5], Izawa [6] and, in a more general framework, Teply and Torrecillas [10]. Some of the present results also generalise for an arbitrary hereditary torsion theory properties established in [2] in the case of the Dickson torsion theory [4].

Now let us give some basic notation and terminology. Throughout the paper we denote by $R$ and $S$ associative rings with non-zero identity and all modules are left unital $R$-modules. If $B$ is a non-empty subset of a module $A$ and $I$ is a non-empty subset of $R$, we denote annihilators as follows:

$$
\begin{aligned}
\operatorname{Ann}_{R} B & =\{r \in R \mid r b=0, \forall b \in B\} \quad \text { and } \\
\operatorname{Ann}_{A} I & =\{a \in A \mid r a=0, \forall r \in I\} .
\end{aligned}
$$

Also, $\tau$ will always be a hereditary torsion theory in the category $R$-Mod of left $R$ modules. For a left $R$-module $A$ and a submodule $B$ of $A, B$ is called $\tau$-dense (respectively $\tau$-closed) in $A$ if $A / B$ is $\tau$-torsion (respectively $\tau$-torsionfree). A non-zero left $R$-module

Received 21st August, 2006

The second author acknowledges the support of the grant CEEX-ET 47/2006.

Copyright Clearance Centre, Inc. Serial-fee code: 0004-9727/07 \$A2.00+0.00. 
module $A$ is said to be $\tau$-cocritical if $A$ is $\tau$-torsionfree and each of its non-zero submodules is $\tau$-dense in $A$. A left $R$-module $A$ is said to be $\tau$-injective if it is injective with respect to every monomorphism having a $\tau$-torsion cokernel or, equivalently, if it is injective with respect to every monomorphism $I \rightarrow R$ with $I$ a $\tau$-dense left ideal of $R$. Any ring homomorphism $\gamma: R \rightarrow S$ allows us to induce in the category $S$-Mod a torsion theory $\sigma$ given by the condition that a left $S$-module $A$ is $\sigma$-torsion if and only if $A$ is $\tau$-torsion as a left $R$-module. Throughout $\sigma$ will always be this induced torsion theory in the corresponding category $S$-Mod. In the above context, $\tau$ is called compatible with $\gamma$ if the following condition holds: a left $S$-module $A$ is $\sigma$-torsionfree if and only if $A$ is $\tau$-torsionfree as a left $R$-module.

For additional information on torsion theories the reader is referred to [5].

\section{FROM $R$ TO $S$}

We begin with the torsion-theoretic generalisation of the result mentioned in the introduction.

THEOREM 2.1. Let $\gamma: R \rightarrow S$ be a ring epimorphism and let $A$ be a $\tau$-injective left $R$-module. Then $\operatorname{Ann}_{A} \operatorname{Ker}(\gamma)$ is $\sigma$-injective as a left $S$-module.

Proof: Denote $K=\operatorname{Ker}(\gamma)$. Let $J$ be a $\sigma$-dense left ideal of $S$ and let $f: J$ $\rightarrow \operatorname{Ann}_{A} K$ be an $S$-homomorphism. Since $\tau$ is compatible with $\gamma$ by [5, Proposition 47.4], $I=\gamma^{-1}(J)$ is a $\tau$-dense left ideal of $R$ [5, Proposition 47.2]. Consider the following diagram with commutative square:

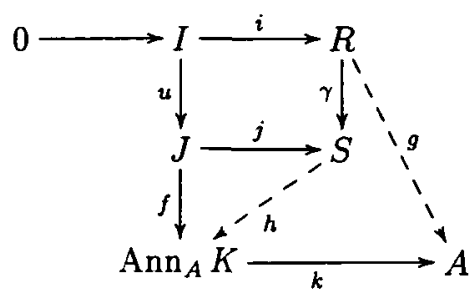

where $i, j, k$ are inclusion homomorphisms and $u$ is the natural $R$-epimorphism induced by $\gamma$. By the $\tau$-injectivity of $A$ as a left $R$-module, there exists an $R$-homomorphism $g: R \rightarrow A$ such that $g i=k f u$. Note that $K \subseteq I$ and $K \subseteq \operatorname{Ker}(g)$.

Now define the $S$-homomorphism

$$
h: S \rightarrow \operatorname{Ann}_{A} K, \quad h(s)=g(r),
$$

where $r \in R$ is such that $\gamma(r)=s$. If $s=\gamma(r)=\gamma\left(r^{\prime}\right)$ for some $r, r^{\prime} \in R$, then $r-r^{\prime} \in K \subseteq \operatorname{Ker}(g)$, hence $g(r)=g\left(r^{\prime}\right)$. If $t \in K$ and $r \in R$, we have $t g(r)=g(t r)=0$, hence $g(r) \in \operatorname{Ann}_{A} K$. Therefore $h$ is well-defined. 
For every $s \in J$, we have $s=u(r)$ for some $r \in I$ and

$$
h j(s)=h j u(r)=h \gamma i(r)=g(i(r))=k f u(r)=k f(s)=f(s) .
$$

Therefore $\operatorname{Ann}_{A} K$ is $\sigma$-injective as a left $S$-module.

Now we can transfer torsion-theoretic injectivity from $R$ to $S$ under the action of a ring epimorphism $\gamma: R \rightarrow S$.

THEOREM 2.2. Let $\gamma: R \rightarrow S$ be a ring epimorphism and let $A$ be a left $R$ module such that $\operatorname{Ker}(\gamma) \subseteq \operatorname{Ann}_{R} A$. If $A$ is $\tau$-injective as a left $R$-module, then $A$ is $\sigma$-injective as a left $S$-module.

Proof: If $\operatorname{Ker}(\gamma) \subseteq \operatorname{Ann}_{R} A$, then $A$ is a left $S$-module and $\operatorname{Ann}_{A} \operatorname{Ker}(\gamma)=A$. Now the result follows by Theorem 2.1 .

\section{From $S$ TO $R$}

We begin with a result in a more general setting, which slightly generalises [10, Proposition 2.7]. It has a similar proof, that we briefly give here for the sake of completeness.

Theorem 3.1. Let $\mathcal{C}$ and $\mathcal{C}^{\prime}$ be two Abelian categories, $\mathcal{T}$ a torsion class in $\mathcal{C}$ and $\mathcal{T}^{\prime}$ a torsion class in $\mathcal{C}^{\prime}$. Also let $G: \mathcal{C} \rightarrow \mathcal{C}^{\prime}$ be an additive functor and $F: \mathcal{C}^{\prime} \rightarrow \mathcal{C}$ an additive functor which preserves the exactness of every short exact sequence $0 \rightarrow X$ $\rightarrow Y \rightarrow Z \rightarrow 0$ with $Z \in \mathcal{T}^{\prime}$. Furthermore, assume that $G$ is a right adjoint to $F$ and $F\left(\mathcal{T}^{\prime}\right) \subseteq \mathcal{T}$. Then $G(A)$ is a $\mathcal{T}^{\prime}$-injective object in $\mathcal{C}^{\prime}$ for every $\mathcal{T}$-injective object $A$ in $\mathcal{C}$.

Proof: Let $A$ be a $\mathcal{T}$-injective object in $\mathcal{C}$ and let $0 \rightarrow X \rightarrow Y \rightarrow Z \rightarrow 0$ be a short exact sequence of objects in $\mathcal{C}^{\prime}$ with $Z \in \mathcal{T}^{\prime}$. Then we have an induced short exact sequence

$$
0 \rightarrow \operatorname{Hom}_{\mathcal{C}}(F(Z), A) \rightarrow \operatorname{Hom}_{\mathcal{C}}(F(Y), A) \rightarrow \operatorname{Hom}_{\mathcal{C}}(F(X), A) \rightarrow 0
$$

and, by adjointness, a short exact sequence

$$
0 \rightarrow \operatorname{Hom}_{\mathcal{C}^{\prime}}(Z, G(A)) \rightarrow \operatorname{Hom}_{\mathcal{C}^{\prime}}(Y, G(A)) \rightarrow \operatorname{Hom}_{\mathcal{C}^{\prime}}(X, G(A)) \rightarrow 0
$$

Then it follows that $G(A)$ is $\mathcal{T}^{\prime}$-injective.

Recall that a right $R$-module $C$ is called $\tau$-flat if the left $R$-module $\operatorname{Hom}_{\mathbb{Z}}(C, \mathbb{Q} / \mathbb{Z})$ is $\tau$-injective or, equivalently, the functor $C \bigotimes_{R}$ - preserves the exactness of every short exact sequence $0 \rightarrow X \rightarrow Y \rightarrow Z \rightarrow 0$ of left $R$-modules with $X$ a $\tau$-dense submodule of $Y$ (see $[5$, p.88] or $[7])$.

COROLLARY 3.2. Let $\gamma: R \rightarrow S$ be a ring homomorphism such that $S$ is $\tau$-flat as a right $R$-module. Also, assume that $\tau$ is compatible with $\gamma$. Then every $\sigma$-injective left $S$-module is $\tau$-injective as a left $R$-module. 
Proof: In Theorem 3.1 take $G: S-$ Mod $\rightarrow R$ - Mod to be the forgetful functor, $F=S \bigotimes_{R}-: R-\operatorname{Mod} \rightarrow S-\operatorname{Mod}, \mathcal{T}^{\prime}$ the torsion class in $R$-Mod associated to the torsion theory $\tau$ and $\mathcal{T}$ the torsion class in $S$-Mod associated to the induced torsion theory $\sigma$. Then $G$ is a right adjoint to $F$. By [5, Proposition 47.2], the compatibility of $\tau$ with $\gamma$ assures that for every $\tau$-torsion left $R$-module $Z, F(Z)=S \bigotimes_{R} Z$ is $\tau$-torsion as a left $R$-module, hence $\sigma$-torsion as a left $S$-module. Now the conclusion follows by Theorem 3.1.

In order to establish a characterisation of the condition that every $\sigma$-injective left $S$-module is $\tau$-injective as a left $R$-module, we use some generalisation of purity. To recall some terminology, let $\Omega$ be a class of left $R$-modules and let

$$
0 \longrightarrow A \stackrel{f}{\longrightarrow} B \stackrel{g}{\longrightarrow} C \longrightarrow 0
$$

be a short exact sequence of right $R$-modules. If the map $f \bigotimes_{R} 1_{D}: A \bigotimes_{R} D \rightarrow B \bigotimes_{R} D$ is a monomorphism for every $D \in \Omega$, then the sequence (1) is called $\Omega$-pure [9]. If $A$ is a submodule of $B, f$ is the inclusion monomorphism and the sequence (1) is $\Omega$-pure, then $A$ is said to be an $\Omega$-pure submodule of $B$. The following characterisation will be useful.

Proposition 3.3. ([9, p. 170]) Let $\Omega$ be a class of cyclic left $R$-modules. The following are equivalent:

(i) The sequence (1) is $\Omega$-pure;

(ii) $A J=A \cap B J$ for every left ideal $J$ of $R$ such that $R / J \in \Omega$.

Now let $\Omega^{\prime}$ be the set of all left $R$-modules $R / J$ with $J$ a $\tau$-dense left ideal of $R$.

Theorem 3.4. Let $\gamma: R \rightarrow S$ be a ring epimorphism and let $K=\operatorname{Ker}(\gamma)$. The following are equivalent:

(i) $K$ is an $\Omega^{\prime}$-pure right ideal of $R$;

(ii) $S$ is $\tau$-flat as a right $R$-module;

(iii) Every $\sigma$-injective left $S$-module is $\tau$-injective as a left $R$-module.

Proof: (i) $\Longleftrightarrow$ (ii) By Proposition 3.3 and $[5$, p.89] both conditions are equivalent to $K I=K \cap I$ for every $\tau$-dense left ideal of $R$.

(ii) $\Longrightarrow$ (iii) By Corollary 3.2.

(iii) $\Longrightarrow$ (ii) Use the injective left $S$-module $\operatorname{Hom}_{\mathbf{Z}}(S, \mathbb{Q} / \mathbb{Z})$ and the definition of $\tau$-flatness.

Now we can put together Theorems 2.2 and 3.4 to get the following result.

THEOREM 3.5. Let $\gamma: R \rightarrow S$ be a ring epimorphism and let $A$ be a left $R$ module such that $\operatorname{Ker}(\gamma) \subseteq \operatorname{Ann}_{R} A$ is an $\Omega^{\prime}$-pure right ideal of $R$. Then $A$ is $\tau$-injective as a left $R$-module if and only if it is $\sigma$-injective as a left $S$-module. 


\section{Applications}

For a commutative ring $R$, denote by $\operatorname{Spec}(R)$ the set of all prime ideals of $R$ and let $p \in \operatorname{Spec}(R)$. Following [12, p.83], for each integer $m \geqslant 1$ denote $A_{m}=\operatorname{Ann}_{E(R / p)} p^{m}$, where $E(R / p)$ is the injective hull of $R / p$. Note that $A_{1} \subseteq A_{2} \subseteq \cdots \subseteq A_{m} \subseteq A_{m+1} \subseteq \ldots$ and, if $R$ is Noetherian, $E(R / p)=\bigcup_{m=1}^{\infty} A_{m}[12, \mathrm{p} .83]$.

Corollary 4.1. Let $R$ be commutative and let $p \in \operatorname{Spec}(R)$ be such that $R / p$ is $\tau$-cocritical. Then $A_{1}=E_{\tau}(R / p)$ is $\sigma$-injective as an $R / p$-module, where $E_{\tau}(R / p)$ is the $\tau$-injective hull of $R / p$ and $\sigma$ is the torsion theory induced by $\tau$ in $R / p$-Mod via the natural ring epimorphism $R \rightarrow R / p$.

Proof: By [3, Theorem 2.5], $A_{1}=E_{\tau}(R / p)$. Since $R / p \subseteq \mathrm{Ann}_{E(R / p)} p$, we have $E_{\tau}(R / p) \subseteq \operatorname{Ann}_{E(R / p)} p$, hence $p \subseteq \operatorname{Ann}_{R} E_{\tau}(R / p)$. Now use Theorem 2.2.

CoRollary 4.2. Let $R$ be commutative Noetherian and let $p \in \operatorname{Spec}(R)$ be $\tau$-closed in $R$. Then:

(i) Each $A_{m}$ is $\sigma_{m}$-injective as an $R / p^{m}$-module, where $\sigma_{m}$ is the torsion theory induced by $\tau$ in $R / p^{m}$-Mod via the natural ring epimorphism $R \rightarrow R / p^{m}$.

(ii) The $\tau$-injective hull $E_{\tau}(R / p)$ of $R / p$ is $\sigma$-injective as an $R / p$-module, where $\sigma$ is the torsion theory induced by $\tau$ in $R / p$-Mod via the natural ring epimorphism $R \rightarrow R / p$.

Proof: (i) Since $p$ is $\tau$-closed in $R, A_{m}$ is $\tau$-injective as an $R$-module [3, Theorem 2.10]. Now use Theorem 2.1 .

(ii) By [3, Theorem 2.10], $A_{1}=\operatorname{Ann}_{E(R / p)} p$ is a $\tau$-injective $R$-module. As in the proof of Corollary 4.1, we have $p \subseteq \operatorname{Ann}_{R} E_{\tau}(R / p)$. Now use Theorem 2.2.

Recall that a ring $R$ is called left fully idempotent if every left ideal of $R$ is idempotent. In particular, any von Neumann regular ring is left fully idempotent.

COROLlary 4.3. Let $R$ be a left fully idempotent ring and let $\gamma: R \rightarrow S$ be a ring epimorphism. Then every $\sigma$-injective left $S$-module is $\tau$-injective as a left $R$-module.

Proof: By [11, p.320], $S$ is flat as a right $R$-module. Now use Corollary 3.2 .

Let us particularise Theorem 3.4 in the case of the Dickson torsion theory $\tau_{D}$, which is generated by all simple left $R$-modules [4]. Then we may restrict $\Omega^{\prime}$ to all simple left $R$-modules and $\Omega^{\prime}$-purity becomes $s$-purity. Recall that a short exact sequence of right $R$-modules is called $s$-pure if it stays exact when tensored by any simple left $R$-module [1]. We denote by $\sigma_{D}$ the torsion theory induced by $\tau_{D}$ in $S$-Mod under the action of a ring homomorphism $\gamma: R \rightarrow S$. Thus we obtain the following result.

COROLlary 4.4. Let $\gamma: R \rightarrow S$ be a ring epimorphism and let $K=\operatorname{Ker}(\gamma)$. The following are equivalent:

(i) $K$ is an $s$-pure right ideal of $R$; 
(ii) $S$ is $\tau_{D}$-flat as a right $R$-module;

(iii) Every $\sigma_{D}$-injective left $S$-module is $\tau_{D}$-injective as a left $R$-module.

Corollary 4.5. Let $\gamma: R \rightarrow S$ be a ring epimorphism such that $K=\operatorname{Ker}(\gamma)$ is an idempotent ideal. Suppose that either $R$ is commutative or $K$ is contained in the Jacobson radical of $R$. Then every $\sigma_{D}$-injective left $S$-module is $\tau_{D}$-injective as a left $R$-module.

ProOF: If either $R$ is commutative or $K$ is contained in the Jacobson radical of $R$, then $K$ is an s-pure ideal of $R$ by [1, Proposition 3.6] and [1, Corollary 2.8]. Now the result follows by Corollary 4.4 .

Now assume that $\tau$ is a Jansian torsion theory, that is, it has a Gabriel filter consisting of all left ideals $J$ of $R$ containing an idempotent two-sided ideal $K$ of $R$. Hence $\Omega^{\prime}=\Omega_{0}$ consists of all left $R$-modules $R / I$ with $I$ a left ideal of $R$ such that $K \subseteq I$. We may characterise idempotent ideals in terms of some purity.

LEMMA 4.6. Let $K$ be a two-sided ideal of $R$. Then $K$ is idempotent if and only if $K$ is $\Omega_{0}$-pure as a right ideal of $R$.

Proof: Assume first that $K$ is idempotent. Let $I$ be a left ideal of $R$ such that $R / I \in \Omega_{0}$. Then $K=K^{2} \subseteq K I \subseteq K \cap I=K$. Hence $K I=K \cap I$, so that $K$ is $\Omega_{0}$-pure in $R$.

Conversely, if $K$ is $\Omega_{0}$-pure in $R$, then we have $K I=K \cap I=K$ for every left ideal $I$ of $R$ such that $R / I \in \Omega_{0}$. In particular, $K^{2}=K$.

COROLlary 4.7. Let $\gamma: R \rightarrow S$ be a ring epimorphism and suppose that $K=\operatorname{Ker}(\gamma)$ is an idempotent ideal. Let $\tau$ be the Jansian torsion theory having the Gabriel filter given by $K$. Then every $\sigma$-injective left $S$-module is $\tau$-injective as a left $R$-module.

Proof: From the proof of Lemma 4.6, we see that the property that $K$ is idempotent is characterised by the condition $K I=K$ for every left ideal $I$ of $R$ such that $R / I \in \Omega_{0}$, that is, for every $\tau$-dense left ideal $I$ of $R$. Also note that one always has $K \subseteq I$, hence $K I=K \cap I$, for any $\tau$-dense left ideal $I$ of $R$. Now use Theorem 3.4.

\section{REFERENCES}

[1] I. Crivei, 's-pure submodules', Internat. J. Math. Math. Sciences 2005 (2005), 491-497.

[2] S. Crivei, 'Injective modules relative to the Dickson torsion theory', Vietnam J. Math. 29 (2001), 329-338.

[3] S. Crivei, ' $\tau$-injective submodules of indecomposable injective modules', J. Korean Math. Soc. 43 (2006), 65-76.

[4] S.E. Dickson, 'A torsion theory for abelian categories', Trans. Amer. Math. Soc. 121 (1966), 223-235. 
[5] J.S. Golan, Torsion theories (Longman Scientific and Technical, New York, 1986).

[6] T. Izawa, 'Torsion theories and torsionless generators', Rep. Fac. Sci. Shizuoka Univ. 11 (1976), 1-8.

[7] H. Katayama, 'Flat and projective properties of a torsion theory', Res. Rep. Ube Tech. Coll. 15 (1972), 1-4.

[8] D.W. Sharpe and P. Vámos, Injective modules (Cambridge University Press, London, New York, 1972).

[9] B. Stenström, 'Pure submodules', Ark. Math. 7 (1967), 151-171.

[10] M. Teply and B. Torrecillas, 'Weak Baer modules over graded rings', Colloq. Math. 75 (1998), 19-31.

[11] R. Wisbauer, Foundations of module and ring theory (Gordon and Breach, Reading, 1991).

[12] J. Xu, Flat covers of modules (Springer-Verlag, Berlin, 1996)).

Department of Mathematics

Technical University

Str. C. Daicoviciu 15

400020 Cluj-Napoca

Romania

e-mail: crivei@math.utcluj.ro
Faculty of Mathematics and Computer Science "Babeş-Bolyai" University

Str. M. Kogălniceanu 1 400084 Cluj-Napoca

Romania

e-mail: crivei@math.ubbcluj.ro

Faculty of Mathematics and Computer Science "Babes-Bolyai" University

Str. M. Kogălniceanu 1

400084 Cluj-Napoca

Romania

e-mail: purdea@math.ubbcluj.ro 